BNL -45517

AD/RHIC-92

Conference Paper

\title{
Beta Functions in the Presence of Linear Coupling
}

\author{
G. Parzen
}

May 1991

\section{R H I C P R O J E C T}

\author{
Brookhaven National Laboratory \\ Associated Universities, Inc. \\ Upton, NY 11973
}




\title{
1991 IEEE Particle Accelerator Conference \\ San Francisco, CA \\ May 6-9, 1991
}

\section{BETA FUNCTIONS IN THE PRESENCE OF LINEAR COUPLING*}

\author{
G. Parzen \\ Brookhaven National Laboratory \\ Upton, NY 11973, USA
}

\section{INTRODUCTION}

One effect of random skew quadrupole field errors is to perturb the $\beta$-functions. This effect may be large in proton accelerators using superconducting magnets, because of the relatively large random skew quadrupole field errors that are expected in these magnets. The effect is also increased by the required insertions in proton colliders which generate large $\beta$-functions in the insertion region.

This effect has been studied in the RHIC accelerator (the Relativistic Heavy Ion Collider proposed at Brookhaven National Laboratory). For RHIC, large changes in the $\beta$-functions were found, as large as $100 \%$ increase in the $\beta$-function in one worse case.

An analytic result has been found for the changes in the beta functions caused by the random $a_{1}$. This result indicates that the important harmonics of $a_{1}$ that need to be controlled are the harmonics near $\nu_{x}+\nu_{y}$. This has been verified in computer studies. The random $a_{1}$ will also generate a large tune splitting $[1,2]$ in RHIC. The analytic result indicates that the correction of the tune splitting will also correct a large part of the errors in the beta functions. This has been shown in computer studies. The $a_{1}$ correction system that has been developed to correct the tune splitting in RHIC appears able to correct most of the error in the beta functions.

\section{RESULTS FOR THE $\beta$-FUNCTIONS}

The random quadrupole field errors expected in RHIC[2] are used in this study. The effect of the random $a_{1}$, the skew quadrupole error, is to couple the $x$ and $y$ motions. The $x$ motion and the $y$ motion can each be written as the sum of 2 normal modes which have the $\nu$-values $\nu_{1}$ and $\nu_{2}$. Each of the normal modes also have $\beta$-functions denoted by $\beta_{1}$ and $\beta_{2}$. For a given distribution of field errors, $\beta_{1}$ and $\beta_{2}$ can be computed using the results of Edwards and Teng.[3]

An analytic result has been found for the change in $\beta_{1}$ and $\beta_{2}$ which is valid close to the resonance $\nu_{x}-\nu_{y}=p$, $p$ being some integer. Let $\beta_{1}$ be the beta function that approaches $\beta_{x}$, the unperturbed beta function, when $a_{1} \rightarrow 0$, and similarly for $\beta_{2}$ and $\beta_{y}$. Then $\left(\beta_{1}-\beta_{x}\right) / \beta_{x}$

\footnotetext{
"Work performed under the auspices of the U.S. Department of Energy.
}

and $\left(\beta_{2}-\beta_{y}\right) / \beta_{y}$ are given by

$$
\begin{gathered}
\left(\beta_{1}-\beta_{x}\right) / \beta_{x}=\sum_{n}\left[\left(\frac{\nu_{1}-\nu_{x}}{\Delta \nu\left(\nu_{1}, \nu_{1}-p\right)}\right) b_{n} \frac{\exp \left[i(n-p) \theta_{x}\right]}{n+\nu_{x}+\nu_{y}}+c . c .\right] \\
\left(\beta_{2}-\beta_{y}\right) / \beta_{y}=\sum_{n}\left[\left(\frac{\nu_{2}-\nu_{y}}{\Delta \nu\left(\nu_{2}+p, \nu_{2}\right)}\right) c_{n} \frac{\exp \left[i(n+p) \theta_{y}\right]}{n+\nu_{x}+\nu_{y}}+c . c .\right] \\
b_{n}=(1 / 4 \pi \rho) \int d s a_{1}\left(\beta_{x} \beta_{y}\right)^{\frac{1}{2}} \exp \left[i\left(-\left(n+\nu_{y}\right) \theta_{x}+\nu_{y} \theta_{y}\right)\right] \\
c_{n}=(1 / 4 \pi \rho) \int d s a_{1}\left(\beta_{x} \beta_{y}\right)^{\frac{1}{2}} \exp \left[i\left(-\left(n+\nu_{x}\right) \theta_{y}+\nu_{x} \theta_{x}\right)\right] \\
\Delta \nu\left(\nu_{x}, \nu_{y}\right)=(1 / 4 \pi \rho) \int d s a_{1}\left(\beta_{x} \beta_{y}\right)^{\frac{1}{2}} \exp \left[i\left(-\nu_{x} \theta_{x}+\nu_{y} \theta_{y}\right)\right] \\
\dot{\theta}_{x}=\psi_{x} / \nu_{x}, \theta_{y}=\psi_{y} / \nu_{y}
\end{gathered}
$$

$\nu_{x}$ and $\nu_{y}$ are the unperturbed tunes. The above results will be derived in a future paper. Eqs. (1) can be written in integral form as

$$
\begin{aligned}
& \left(\beta_{1}-\beta_{x}\right) / \beta_{x}=-\frac{\nu_{1}-\nu_{x}}{\left|\Delta \nu\left(\nu_{1}, \nu_{1}-p\right)\right|} \frac{1}{2 \rho \sin \pi\left(\nu_{x}+\nu_{y}\right)} \\
& \quad \times \int d s^{\prime} a_{1}\left(s^{\prime}\right)\left(\beta_{x}\left(s^{\prime}\right) \beta_{y}\left(s^{\prime}\right)\right)^{\frac{1}{2}} \\
& \cos \left[ \pm \pi\left(\nu_{x}+\nu_{y}\right)-\left(\nu_{x}+\nu_{y}\right)\left(\theta-\theta^{\prime}\right)+\nu_{y}\left(\theta_{y}^{\prime}-\theta_{x}^{\prime}\right)-\delta_{1}\right]
\end{aligned}
$$

The integral form for $\left(\beta_{2}-\beta_{y}\right) / \beta_{y}$ is obtained from $\mathrm{Eq}$. (2) through the substitutions in Eq. (2) of $\beta_{1} \rightarrow \beta_{2}$, $\beta_{x} \rightarrow \beta_{y}, \nu_{x} \rightarrow \nu_{y}, \nu_{y} \rightarrow \nu_{x}, \delta_{1} \rightarrow \delta_{2}, \Delta \nu\left(\nu_{1}, \nu_{1}-p\right) \rightarrow$ $\Delta \nu\left(\nu_{2}+p, \nu_{2}\right), \nu_{1} \rightarrow \nu_{2}, \delta_{1}=$ phase $\left[\Delta \nu\left(\nu_{1}, \nu_{1}-p\right)\right]$ and $\delta_{2}=$ phase $\left[\Delta \nu^{*}\left(\nu_{2}+p, \nu_{2}\right)\right]$. For the \pm , the top sign is used for $\theta>\theta^{\prime}$ and the bottom sign for $\theta<\theta^{\prime}$.

Eq. (1) shows that the important harmonics in $a_{1}$ are the harmonics near $\nu_{x}+\nu_{y}$, and a correction system for $\beta_{1}, \beta_{2}$ should probably control the harmonics of $a_{1}$ near $\nu_{x}+\nu_{y}$. However, Eq. (1) shows that the dominant harmonic in $\left(\beta_{1}-\beta_{x}\right) / \beta_{x}$ is the $2 \nu_{x}$ harmonic and in $\left(\beta_{2}-\beta_{y}\right) / \beta_{y}$ the $2 \nu_{y}$ harmonic. Near the $\nu_{x}-\nu_{y}=0$ resonance, the $2 \nu_{x}, 2 \nu_{y}$ and $\nu_{x}+\nu_{y}$ harmonics are about the same. However they are different near the $\nu_{x}-\nu_{y}=p$ resonance. The above formulae appear to suggest the effects of a $\nu_{x}+\nu_{y}=$ integer sum resonance. However it may be more proper to label it as a half-integer resonance effect in the coordinate system of the normal modes.

One may note the factor $\left(\nu_{1}-\nu_{2}\right) / \Delta \nu$. For large $\Delta \nu, \Delta \nu>\left(\nu_{x}-\nu_{y}-p\right)$, then this function approaches 1. This may be seen from the result for $\nu_{1}$ and $\nu_{2}$ near 
the $\nu_{x}-\nu_{y}=0$ resonance.

$$
\begin{aligned}
& \nu_{1}=\bar{\nu} \pm\left[\left(\nu_{x}-\nu_{y}\right)^{2} / 4+|\Delta \nu|^{2}\right]^{\frac{1}{2}}, \\
& \nu_{2}=\bar{\nu} \mp\left[\left(\nu_{x}-\nu_{y}\right)^{2} / 4+|\Delta \nu|^{2}\right]^{\frac{1}{2}}, \\
& \bar{\nu}=\left(\nu_{x}+\nu_{y}\right) / 2, \quad \Delta \nu=\Delta \nu(\bar{\nu}, \bar{\nu}) .
\end{aligned}
$$

For the \pm , the top sign is used for $\nu_{x}>\nu_{y}$ and the bottom sign for $\nu_{x}<\nu_{y}$. According to Eq. (3) $\left(\nu_{1}-\nu_{x}\right) / \Delta \nu \rightarrow 1$ for large $\Delta \nu$ and $\left(\nu_{1}-\nu_{x}\right) / \Delta \nu \simeq 2 \Delta \nu /\left|\left(\nu_{x}-\nu_{y}\right)\right|$ for small $\Delta \nu$. For very small $\Delta \nu$, the result is not easy to compute correctly because of higher order term in $\alpha_{1}$ that are not given in Eqs. (3). Computer studies show that correction of $\nu_{1}$ and $\nu_{2}$ to make them closer to $\nu_{x}, \nu_{y}$ tends to decrease $\left(\beta_{1}-\beta_{x}\right) / \beta_{x}$ and $\left(\beta_{2}-\beta_{y}\right) / \beta_{y}$ which may be due in part to this $\left(\nu_{1}-\nu_{x}\right) / \Delta \nu$ factor.

Table 1: Maximum $\beta_{1}, \beta_{2}$ for 10 distributions of random $a_{1}$ fields for the RHIC $\beta^{*}=2$ lattice. No corrections are present.

\begin{tabular}{|c|c|c|}
\hline $\begin{array}{c}\text { Error } \\
\text { Field }\end{array}$ & $\begin{array}{c}\beta_{1, \max } \\
\text { at QF } \\
(\mathrm{m})\end{array}$ & $\begin{array}{c}\beta_{2, \max } \\
\text { at QD } \\
(\mathrm{m})\end{array}$ \\
\hline 1 & 68 & 75 \\
2 & 138 & 95 \\
3 & 89 & 78 \\
4 & 83 & 74 \\
5 & 69 & 65 \\
6 & 65 & 67 \\
7 & 76 & 82 \\
8 & 78 & 79 \\
9 & 80 & 78 \\
10 & 77 & 87 \\
\hline
\end{tabular}

One may also note that $\left(\beta_{1}-\beta_{x}\right) / \beta_{x}$ is linear in $a_{1}$ for large $\Delta \nu$, and quadratic in $a_{1}$ for small $\Delta \nu$.

A result for the rms value of $\left(\beta_{1}-\beta_{x}\right) / \beta_{x}$ due to random distribution of $a_{1}$ errors may be obtained from the integral form $\mathrm{Eq}$. (2), for the case when $|\Delta \nu|>>\left|\nu_{x}-\nu_{y}-p\right|$. In this case $\left|\nu_{1}-\nu_{x}\right| /|\Delta \nu| \simeq 1$ and

$$
\begin{aligned}
& \left(\frac{\beta_{1}-\beta_{x}}{\beta_{x}}\right)_{r m s}^{2}=\sum_{k}\left(\frac{\beta_{1}-\beta_{x}}{\beta_{x}}\right)_{k, r m s}^{2} \\
& \left(\frac{\beta_{1}-\beta_{x}}{\beta_{x}}\right)_{k, r m s}=N_{k}^{1 / 2} \frac{\left(\left(\beta_{x} \beta_{y}\right)^{1 / 2} a_{1, r m s}\right)_{k}}{2.8 \rho \sin \pi\left(\nu_{x}+\nu_{y}\right)}
\end{aligned}
$$

where the index $k$ indicates the different types of magnets. $N_{k}$ is the number of magnets of a certain type. Eq. (4) also gives the result for $\left(\left(\beta_{2}-\beta_{y}\right) / \beta_{y}\right)_{r m s}$. One also sees that

$$
\left(\left(\beta_{1}-\beta_{x}\right) / \beta_{x}\right)_{r m s}=\left[4 \pi /\left(2.8 \sin \pi\left(\nu_{x}+\nu_{y}\right)\right)\right] \Delta \nu_{r m s}
$$

where $\Delta \nu_{r m s}$ is the rms value of $\Delta \nu$.
Table 2: Maximum $\beta_{1}$ at $\mathrm{QF}$ and the maximum $\beta_{2}$ at $\mathrm{QD}$ for 10 distributions of random $a_{1}$ fields, for the RHIC $\beta^{*}=2$ lattice, showing the effect of correcting the tune splitting on $\beta_{1}, \beta_{2}$.

\begin{tabular}{|c|c|c|c|c|}
\hline \multirow{2}{*}{$\begin{array}{c}\text { Error } \\
\text { Field }\end{array}$} & \multicolumn{2}{|c|}{$\begin{array}{c}2 \text { Family } \\
\text { Correction }\end{array}$} & \multicolumn{2}{c|}{$\begin{array}{c}\text { Enlarged } a_{1} \\
\text { Correction } \\
\text { System }\end{array}$} \\
& $\beta_{1, \max }$ & $\beta_{2, \max }$ & $\beta_{1, \max }$ & $\beta_{2, \max }$ \\
\hline 1 & 58 & 58 & 57 & 56 \\
2 & 57 & 54 & 59 & 74 \\
3 & 56 & 59 & 56 & 59 \\
4 & 63 & 57 & 61 & 56 \\
5 & 63 & 60 & 63 & 60 \\
6 & 55 & 58 & 58 & 62 \\
7 & 102 & 84 & 61 & 59 \\
8 & 60 & 57 & 64 & 60 \\
9 & 58 & 59 & 58 & 58 \\
10 & 60 & 78 & 57 & 56 \\
\hline
\end{tabular}

Table 1 lists the beta functions, before any correction of the random $a_{1}$ fields for the RHIC $\beta^{*}=2$ lattice for ten distributions of the random $a_{1}$. The table lists the maximum $\beta_{1}$ found at the normal focusing quadrupoles, $\mathrm{QF}$, and the maximum $\beta_{2}$ at the normal defocusing quadrupoles, $\mathrm{QD}$. The unperturbed value of the maximum $\beta_{x}$ and $\beta_{y}$ is $50 \mathrm{~m}$. One sees that change in the beta functions of the order of $100 \%$ are computed. $\beta_{1}$ and $\beta_{2}$ were computed using the exact results of Edwards and Teng.[3]

Using Eq. (4), one can compute the expected rms value of $\left(\beta_{1}-\beta_{x}\right) / \beta_{x}$ which gives for RHIC $\left(\left(\beta_{1}-\beta_{x}\right)\right.$ $\left./ \beta_{x}\right)_{r m s}=0.29$. For 72 QF magnets, the $90 \%$ probability result is $\beta_{1, \text { max }}=\beta_{2, \text { max }}=95$. This is in fairly good agreement with the results in Table 1 . The exceptionally large $\beta_{1, \text { max }}=138$ for seed 2 is due to $\nu_{1}$ being shifted close to the $\nu_{1}=29$ resonance where $\beta_{1}$ would become infinite. This effect of the $\nu_{1}=29$ resonance is not included in our results for $\left(\beta_{1}-\beta_{x}\right) / \beta_{x}$.

\section{CORRECTION OF $\beta_{1}, \beta_{2}$}

Computer studies indicate that when the tune shifts $\nu_{1}-\nu_{x}$ and $\nu_{2}-\nu_{y}$ are corrected, then $\beta_{1}-\beta_{x}$ and $\beta_{2}-\beta_{y}$ are also reduced. This is indicated by the analytical result Eqs. (1). It is partly due to the $\left(\nu_{1}-\nu_{x}\right) / \Delta \nu$, $\left(\nu_{2}-\nu_{y}\right) / \Delta \nu$ factors in Eqs. (1). It is also partly due to the driving terms $b_{n}, c_{n}$ in Eq. (1), which are also important driving terms for the tune splitting.[4] In RHIC the tune splitting correction includes a 2 family $a_{1}$ correction system, that corrects the different resonance, $\nu_{x}-\nu_{y}=0$, and most of the tune splitting, and an enlarged $a_{1}$ correction system[4] to correct the residual tune splitting by controlling the harmonics of $a_{1}$ near $\nu_{x}+\nu_{y}$.

Table 2 shows what happens to $\beta_{1}, \beta_{2}$ when the tune splitting is corrected by the above 2 family tune splitting correction systems. One sees that the $\beta_{1}-\beta_{x}, \beta_{2}-\beta_{y}$ errors are considerably reduced. The results in Table 2, include the effects of random $b_{1}$ field which can generate 
a $20 \%$ error in $\beta_{1}$ and $\beta_{2}$. However, a considerable error in $\beta_{1}, \beta_{2}$ remains for some distributions.

Table 2 also shows what happens to $\beta_{1}, \beta_{2}$ when the tune splitting is further reduced using the enlarged $a_{1}$ correction system to control harmonics near $\nu_{x}+\nu_{y}$. One sees a considerable improvement in $\beta_{1}$ and $\beta_{2}$.

The results for $\beta_{1}, \beta_{2}$ can be further improved by adjusting the harmonics of $a_{1}$ near $\nu_{x}+\nu_{y}$, or by moving the $\nu$-values slightly closer to the resonance line $\nu_{x}-\nu_{y}=$ 0 . At this level, the correction of the tune splitting and the $\beta_{1}, \beta_{2}$ correction may be competing with each other.
Using these procedures the largest error in $\beta_{1}, \beta_{2}$ was reduced to $28 \%$, which includes the $20 \%$ effect due to the random $b_{1}$ which has not been corrected.

\section{REFERENCES}

[1] G. Parzen, BNL Report AD/RHIC-AP-72 (1988).

[2] G. Parzen, BNL Report AD/RHIC-82 (1990).

[3] D. Edwards and L. Teng, IEEE 1973 PAC, p. 885.

[4] G. Parzen, Tune splitting in the presence of linear coupling, these proceedings. 